\title{
Survey of Redox-Active Moieties for Application in Multiplexed Electrochemical Biosensors
}

\author{
Di Kang, ${ }^{\dagger}$ Francesco Ricci, ${ }^{\dagger}$ Ryan J. White, ${ }^{\S}$ and Kevin W. Plaxco*, ${ }^{\dagger}, \|$
}

${ }^{\dagger}$ Department of Chemistry and Biochemistry and "Interdepartmental Program in Biomolecular Science and Engineering, University of California Santa Barbara, Santa Barbara, California 93106, United States

${ }^{\ddagger}$ Dipartimento di Scienze e Tecnologie Chimiche, University of Rome, Tor Vergata, Via della Ricerca Scientifica, 00133, Rome, Italy

${ }^{\S}$ Department of Chemistry and Biochemistry, University of Maryland, Baltimore County, Baltimore, Maryland 21250, United States

Supporting Information

ABSTRACT: Recent years have seen the development of a large number of electrochemical sandwich assays and reagentless biosensor architectures employing biomolecules modified via the attachment of a redox-active "reporter." Here we survey a large set of potential redox reporters in order to determine which exhibits the best long-duration stability in thiol-on-gold monolayer-based sensors and to identify reporter "sets" signaling at distinct, nonoverlapping redox potentials in support of multiplexing and error correcting ratiometric or differential measurement approaches. Specifically, we have characterized the performance of more than a dozen potential reporters that are, first, redox active within the potential window over which thiol-on-gold monolayers are reasonably stable and, second, are available commercially in forms that are readily conjugated to biomolecules or can be converted into such forms in one or two simple synthetic steps. To test each

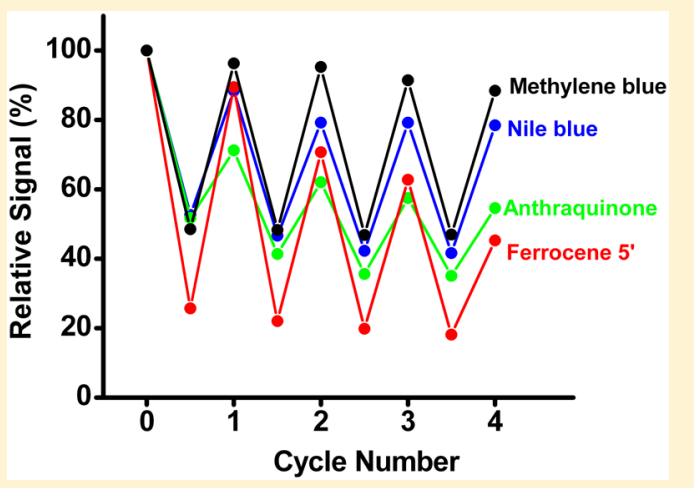
of these reporters we conjugated it to one terminus of a single-stranded DNA "probe" that was attached by its other terminus via a six-carbon thiol to a gold electrode to form an "E-DNA" sensor responsive to its complementary DNA target. We then measured the signaling properties of each sensor as well as its stability against repeated voltammetric scans and against deployment in and reuse from blood serum. Doing so we find that the performance of methylene blue-based, thiol-on-gold sensors is unmatched; the near-quantitative stability of such sensors against repeated scanning in even very complex sample matrices is unparalleled. While more modest, the stability of sensors employing a handful of other reporters, including anthraquinone, Nile blue, and ferrrocene, is reasonable. Our work thus serves as both to highlight the exceptional properties of methylene blue as a redox reporter in such applications and as a cautionary tale-we wish to help other researchers avoid fruitless efforts to employ the many, seemingly promising and yet ultimately inadequate reporters we have investigated. Finally, we hope that our work also serves as an illustration of the pressing need for the further development of useful redox reporters.

$\mathrm{D}$ ue to the ease with which they are fabricated and employed and their oft-impressive selectivity and detection limits, electrochemical biosensors employing redoxreporter-modified oligonucleotides ${ }^{1}$ and polypeptides ${ }^{2}$ attached via thiol self-assembled monolayer (SAM) formation onto gold electrodes have seen significant recent attention. These include analytical approaches ranging from sandwich ${ }^{3}$ and intercalation assays $^{4}$ to reagentless sensors based on binding-induced conformational changes (reviewed in ref 1) in collision efficiency ${ }^{5}$ or changes in through-DNA charge transfer. ${ }^{4,6}$ The potential advantages of such sensors are multifold. First, many perform well even when deployed directly in complex clinical samples, such as undiluted blood serum, saliva, or crude cell lysates (e.g., refs 7-9), and have even been used for the continuous measurement of plasma drug levels. ${ }^{10}$ Second, the approach is quite convenient, with sensor fabrication generally proving facile (e.g., ref 11) and the electronics required to interrogate them being small and inexpensive. ${ }^{12}$
Beyond the above attributes, an additional advantage of SAM-on-gold-based electrochemical biosensors is the ability to deploy multiple sensors on a single electrode by employing reporters that signal at unique, nonoverlapping reduction potentials. This allows for improved multiplexing (e.g., refs 13 and 14) and for the introduction of error correcting, ratiometric or differential measurement approaches (e.g., refs 15 and 16). To date, however, the literature describing these sensors has almost entirely utilized methylene blue, ${ }^{6}$ Nile blue, anthraquinone, or ferrocene as reporters (e.g., refs 6 and 17-21). The expansion of this relatively short list could improve the extent to which such sensors can be multiplexed. Moreover, we have found that a lack of redox reporters of similar stability hinders ratiometric and differential error correction methods, which

Received: June 20, 2016

Accepted: September 23, 2016

Published: September 23, 2016 
suffer if one reporter degrades more rapidly than the other. Motivated by these observations we have, over many years of work in this area, characterized more than a dozen candidate redox reporters (Figure 1), all of which are either commercially

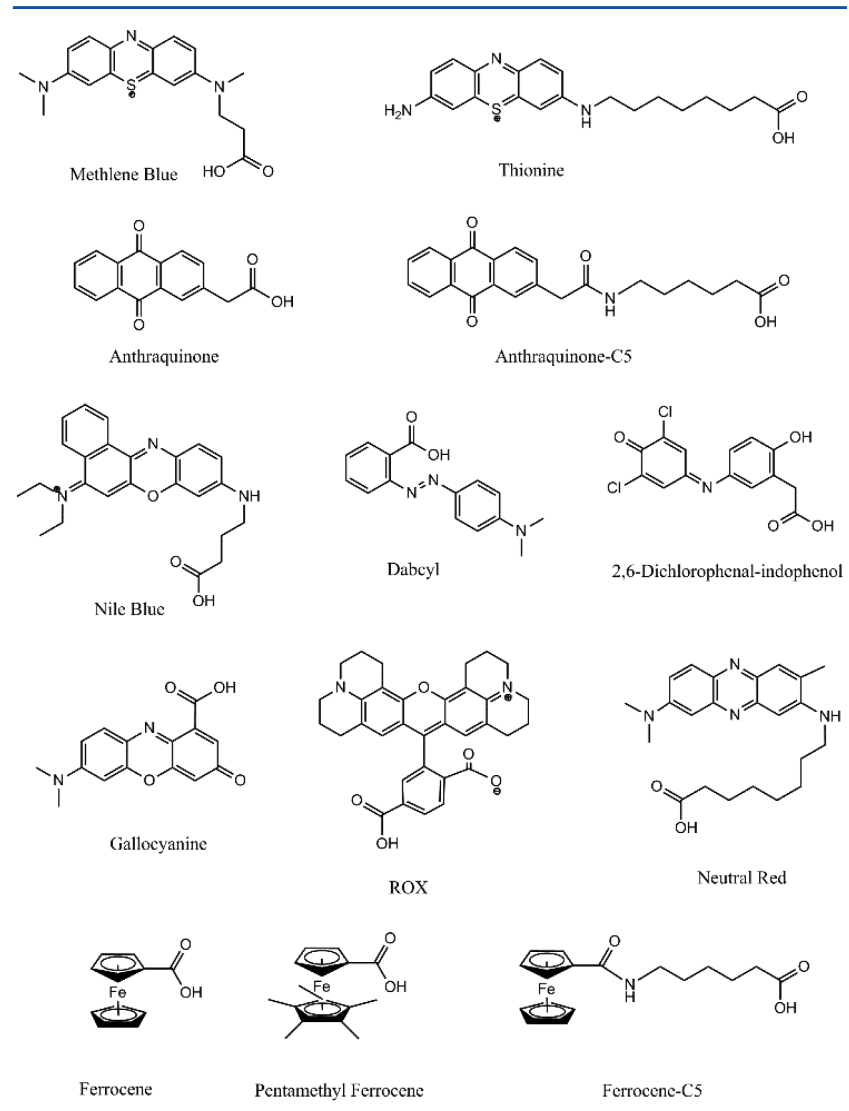

Figure 1. We characterized 13 potential redox reporters in total, 9 organic small molecules, and 2 organometallic complexes. Two of these, ferrocene and anthraquinone, were investigated with and without an additional five-carbon linker and one, ferrocene, was investigated using both $3^{\prime}$ and $5^{\prime}$ linkages. All these potential reporters are available in (or are easily synthesized as) forms containing a carboxylic acid group for ready conjugation to an amine-modified DNA.

available in forms easily conjugated to biomolecules or are readily converted into such forms via one or two simple synthetic steps and all of which are active at potentials within the $\sim 1 \mathrm{~V}$ window over which thiol-on-gold SAMs are reasonable stable. Unfortunately, though, the overwhelming majority of the resulting sensors were unstable thus we abandoned most of these potential reporters without reporting their characterization in the literature. Recently, however, we have become aware that other researchers had similarly characterized and found unsuitable many of the same potential reporters. In order to (1) highlight the exceptional stability of the "best" redox reporter in such applications, (2) identify other reporters that, while nonoptimal, still perform "well enough" for use in ratiometric or multiplexed sensors, (3) help future researchers avoid repeating yet again these same, largely failed stability studies (none of which have previously been published, thus, causing unnecessary repetition of effort), and (4) motivate efforts to identify new redox reporters suitable for use with thiol-on-gold-based sensors, we describe here the results of our decade-plus efforts.

\section{EXPERIMENTAL SECTION}

Reagents. Anhydrous ferrous chloride (Sigma-Aldrich), sodium nitrite (Sigma-Aldrich), sodium cyclopentadienide (Sigma-Aldrich), $n$-butyllithium (1.6 M in $n$-hexane; SigmaAldrich), pentamethylcyclopentadiene (Sigma-Aldrich), 2chlorobenzoyl chloride (Sigma-Aldrich), aluminum chloride (Sigma-Aldrich), potassium $t$-butoxide (Sigma-Aldrich), methyl tertiary butyl ether (Sigma-Aldrich), ferrocene-carboxylic acid, $\mathrm{N}$-hydroxysuccinimide (Sigma-Aldrich), $\mathrm{N}$-hydroxysulfosuccinimide sodium salt (NHS), $N$-(3-dimethylamino-propyl)- $N^{\prime}$ ethylcarbodiimide (Fluka), 2,6-dichloro-p-benzoquinone-4chloroimine (Fisher), 2-hydroxyphenylacetic acid (Fisher), neutral red (Sigma-Aldrich), 3,7-bis ( $\mathrm{N}$-(3-carboxypropyl)- $N$ methylamino)-phenothiazin-5-ium perchlorate (MB -NHS, empBiotech $\mathrm{GmbH}$ ), anthraquinone-NHS (AQ-NHS, empBiotech $\mathrm{GmbH}$ ), anthraquinone-2-amidopentyl carboxylic acid NHS ester (empBiotech $\mathrm{GmbH}$ ), gallocyanine carboxylic acid NHS ester (empBiotech $\mathrm{GmbH}$ ), ferrocene-amidopentyl carboxylic acid NHS ester (empBiotech $\mathrm{GmbH}$ ), phosphate buffer (Sigma-Aldrich), 4-(2-hydroxyethyl)-1-piperazineethanesulfonic acid (HEPES; Sigma-Aldrich), and fetal calf serum (Sigma-Aldrich) were all used as received.

For the majority of our studies we employed this sequence as our E-DNA “probe.”

5'-HS- $\left(\mathrm{CH}_{2}\right)_{6}$-TGGATCGGCGTTTTATT- $\left(\mathrm{CH}_{2}\right)_{6}-\mathrm{NH}_{2}$ $3^{\prime}$, which we conjugated in-house to carboxyl-modified reporters to form an amide bond (see below). Five of the potential reporters we characterized, however, were instead obtained as DNA conjugates directly from a commercial synthesis house (Biosearch, Inc., Novato, CA): 5'-HS- $\left(\mathrm{CH}_{2}\right)_{6}$ TGGATCGGCGTTTTATT- $\left(\mathrm{CH}_{2}\right)_{6}-(\mathrm{NH}-\mathrm{CO})-\mathrm{ROX}-3^{\prime} ; 5^{\prime}$ HS- $\left(\mathrm{CH}_{2}\right)_{6}$-TGGATCGGCGTTTTATT- $\left(\mathrm{CH}_{2}\right)_{6}-(\mathrm{NH}-\mathrm{CO})$ dabcyl-3'; 5'-HS-( $\left.\mathrm{CH}_{2}\right)_{6}$-TGGATCGGCGTTTTATT$\left(\mathrm{CH}_{2}\right)_{6}$ - $(\mathrm{NH}-\mathrm{CO})$-anthraquinone-3'; $5^{\prime}$-HS- $\left(\mathrm{CH}_{2}\right)_{6}$-ATTATTGATCGGCGTTTTAAAGAAG- $\left(\mathrm{CH}_{2}\right)_{6}$-(NH-CO)-methylene blue-3'; 3'-HS-( $\left.\mathrm{CH}_{2}\right)_{6}$-AGACAAGGAAAATCCTTCAATGAAGTGGGTCG- $\left(\mathrm{CH}_{2}\right)_{6}$-(NH-CO)-ferrocene-5'.

As the target olignonucleotide for these sensors, we employed the following unmodified DNA constructs as appropriate: 5'-AATAAAACGCCGATCCA-3'; 5'-TAAAACGCCGATC-3'; 5'-CGACCCACTTCATTGAAGGATTTTCCTTGTCT-3'.

Synthesis of Succinimidyl Ester-Modified Reporters. Thionine. We mixed thionine $(2.6 \mathrm{~g})$ and 8-bromooctanoic acid $(3 \mathrm{~g})$ in DMF $(40 \mathrm{~mL})$ to synthesize $3 \mathrm{~N}$-octanoic-acidmodified thionine. The mixture was refluxed overnight $(10 \mathrm{~h})$. The crude product mixture was concentrated in vacuum, before being dissolved in methanol and filtered (Celite). We use silica column chromatography (100:12:1.2 with chloroform/methanol/acetic acid) to purify the product $(251 \mathrm{mg})$ with poor yield $(7.5 \%)$.

To synthesize thionine succinimidyl ester, we mixed $N, N^{\prime}$ dicyclohexylcarbodiimide (DCC), N-hydrosuccinamide (NHS), and 3N-octanoic-acid-modified thionine in dry dimethylformamide (DMF). The solution was stirred overnight under argon at room temperature. We concentrated the mixture in vacuum, and purified the product with silica column chromatography (100:12:1.2 chloroform/methanol/acetic acid).

Nile Blue. We first prepared 5-(dimethylamino)-2-nitrosophenol by dissolving $826 \mathrm{mg}$ of 3-(dimethylamino)phenol in concentrated hydrochloric acid $(5 \mathrm{~mL})$ at $0{ }^{\circ} \mathrm{C}$, followed by the 
slow addition of $400 \mathrm{mg}$ sodium nitrite, stirring until the content solidified (about $40 \mathrm{~min}$ ). The precipitate was isolated via vacuum filtration and washed with chilled $1 \mathrm{M}$ hydrochloric acid. The crude product, a yellow-brown solid, was dried in vacuum and used for the conjugation reaction without further purification. In parallel, we prepared 3-(naphthalen-1-ylamino) propanoic acid by dissolving $200 \mathrm{mg}$ naphthylamine in DMF $(15 \mathrm{~mL})$ followed by the addition of $5 \mathrm{~mL}$ of $6 \mathrm{M}$ sodium hydroxide and equimolar amounts of dissolved 3-bromopropionic acid ethyl ester. We refluxed the mixture for $12 \mathrm{~h}$. After the reaction finished, we dried the crude reaction mixture in vacuum and purify the product using silica column chromatography (20:1 hexane/methanol). To obtain propionic acid modified Nile blue we first dissolved $34 \mathrm{mg}$ of 5(dimethylamino)-2-nitrosophenol and $44 \mathrm{mg}$ of 3-(naphthalen1-ylamino)propanoic acid in DMF $(10 \mathrm{~mL})$, and heated the reaction mixture to $90{ }^{\circ} \mathrm{C}$ for overnight. We dried the crude reaction mixture in vacuum and purified it using silica column chromatography $\left(3: 1 \mathrm{CHCl}_{3} /\right.$ methanol).

2,6-Dichlorophenal-Indophenol. We dissolved $150 \mathrm{mg}$ 2,6dichloro-p-benzoquinone-4-chloroimine in $2 \mathrm{~mL}$ of methanol and 2-hydroxyphenylacetic acid in $10 \mathrm{~mL}$ of $100 \mathrm{mM} \mathrm{K}_{2} \mathrm{HPO}_{4}$. We then mixed two solutions and stirred overnight at room temperature. The resulting solution was dried under vacuum to produce the crude purple blue product which we purified using silica column chromatography (100:10:1 chloroform/methanol/acetic acid).

Ferrocene. We use the previously described procedure ${ }^{20}$ to convert ferrocene carboxylic acid to ferrocene succinimidyl ester (Fc-NHS). In brief, we mixed a 5-fold excess of NHS and EDC with ferrocene carboxylic acid in dichloromethane (DCM). The solution was stirred overnight under argon $(\sim 12 \mathrm{~h})$ at room temperature. The resulting solution was then washed with water and the organic phase was collected and dried with $\mathrm{MgSO}_{4}$, filtered, and evaporated. Finally, we used silica column chromatography with diethyl ether to purify the product (Fc-NHS).

Pentamethyferrocene. A suspension of $\mathrm{FeCl}_{2}$ in tetrahydrofuran (THF) was vigorously stirred in the dark $1 \mathrm{~h}$ to produce $\mathrm{FeCl}_{2} \cdot \mathrm{THF}$. Separately, $n$-butyllithium was added dropwise to pentamethylcyclopentadiene in tetrahydrofuran in a dry ice acetone bath and the mixture was then warmed and stirred at room temperature for $2 \mathrm{~h}$. We then slowly transferred this mixture into the $\mathrm{FeCl}_{2}$. THF solution and stirred at room temperature for $1 \mathrm{~h}$. Sodium cyclopentadienide was slowly added to this via cannula before stirring overnight $(\sim 16 \mathrm{~h})$. The resultant pentamethylferrocene product was recrystallized from pentane with a yield of $60 \%$.

To obtain pentamethylferrocene carboxylic acid we first mixed 2-chlorobenzoyl chloride and $\mathrm{AlCl}_{3}$ in dichloromethane for $1 \mathrm{~h}$ at $0{ }^{\circ} \mathrm{C}$. We cooled this to $-40^{\circ} \mathrm{C}$ and then slowly add pentamethylferrocene in dichloromethane. We then warmed the mixture to $15{ }^{\circ} \mathrm{C}$ over $60 \mathrm{~min}$ to generate 2-chlorobenzoylpentamethylferrocene which we then poured onto crushed ice. We next washed the organic phase with $1 \mathrm{M} \mathrm{NaOH}$ and dried it against $\mathrm{MgSO}_{4}$ before filtering and evaporating the solvent. We purified the crude 2-chlorobenzoyl-pentamethylferrocene using silica column chromatography (25:1 cyclohexane/methyl tertbutyl ether). We then mixed the 2-chlorobenzoyl-pentamethylferrocene with potassium tert-butoxide and few drops of water in dimethylformamide (DMF) and refluxed the mixture at $110{ }^{\circ} \mathrm{C}$ for $1 \mathrm{~h}$ to produce the carboxylic acid. After cooling the mixture to $0{ }^{\circ} \mathrm{C}$ we then added $1 \mathrm{M} \mathrm{HCl}$ to obtain the red brown solid, which we washed with water, filtered, and dried.

To synthesize pentamethyferrocene succinimidyl ester we added NHS and $N$-(3-dimethylamino-propyl)- $N^{\prime}$-ethylcarbodiimide hydrochloride (EDC) to a $25 \mathrm{mM}$ solution of pentamethylferrocene carboxylic acid in dichloromethane to a final concentration of $60 \mathrm{mM}$ each and stirred overnight $(\sim 12$ h) under argon at room temperature. The resulting solution was then washed with water, and the organic phase was collected and dried with $\mathrm{MgSO}_{4}$, filtered, and evaporated. We then purified the pentamethyferrocene succinimidyl ester product using silica column chromatography (diethyl ether).

Conjugating the Reporters to the DNA Probes. Conjugation of the redox reporters to the appropriate singlestranded DNA was achieved via the coupling of the NHS-ester redox reporter conjugate with the $5^{\prime}$-alkyl-amino modified single stranded DNA. A total of $10 \mu \mathrm{L}$ of $200 \mu \mathrm{M} 5^{\prime}$-alkylamino DNA was added to $50 \mu \mathrm{L}$ of a $0.5 \mathrm{M}$ sodium bicarbonate solution ( $\mathrm{pH} 8.5$ ), and $1 \mu$ mole of the reporter-NHS was dissolved in $10 \mu \mathrm{L}$ dimethyl sulfoxide (DMSO). ${ }^{22}$ We mixed the DNA solution we prepared above and the reporter-NHS DMSO solution, and incubated the mixture for $4 \mathrm{~h}$ in dark at room temperature. After conjugation the DNA was desalted using a spin column (EMP-Biotech) and purified by RP-HPLC (C18 column). The stocked DNA solutions were stored at -20 ${ }^{\circ} \mathrm{C}$ for future use. The yield of the final conjugated product was estimated using HPLC and gel electrophoresis (Figure SI 1). Conveniently, unmodified DNA is not electroactive at the potentials we employed and thus is silent in our experiments.

Electrode Preparation and Sensor Fabrication. E-DNA sensors were prepared using established procedures. ${ }^{10}$ In brief, prior to sensor fabrication, gold disk electrodes $(2 \mathrm{~mm}$ diameter, $\mathrm{CH}$ Instruments, Austin, TX) were cleaned both mechanically (by polishing with diamond and alumina oxide slurries successively) and electrochemically (through successive scans in sulfuric acid solutions) as previously described. The linear probe DNA were reduced for $1 \mathrm{~h}$ at room temperature in $10 \mathrm{mM}$ tris(2-carboxyethyl)phosphine hydrochloride (Molecular Probes, Carlsbad, CA) and then diluted to a final concentration of $1 \mu \mathrm{M}$ in $50 \mathrm{mM}$ phosphate, $100 \mathrm{mM} \mathrm{NaCl}$ buffer, $\mathrm{pH} \mathrm{7.0,} \mathrm{as} \mathrm{was} \mathrm{used} \mathrm{in} \mathrm{all} \mathrm{the} \mathrm{experiments} \mathrm{to} \mathrm{follow}$ unless otherwise noted). The gold electrodes were incubated in this solution for $1 \mathrm{~h}$ at room temperature, rinsed with deionized water, and then incubated in $3 \mathrm{mM}$ 6-mercapto-1-hexanol in deionized water for $120 \mathrm{~min}$. After deposition of this molecule onto a gold electrode the electrode surface is "backfilled" with 6-mercapto-1-hexanol to form a continuous, mixed, selfassembled monolayer. Following this, the electrodes were rinsed in deionized water and stored in buffer for future use.

Sensor Characterization. Fabricated sensors were interrogated using square wave voltammetry (SWV) with a $50 \mathrm{mV}$ amplitude signal at a frequency of $60 \mathrm{~Hz}$, in the absence and presence of fully complementary target. For the latter measurements the electrodes were incubated for $30 \mathrm{~min}$ with the target DNA at $1 \mu \mathrm{M}$ in $50 \mathrm{mM}$ phosphate, $100 \mathrm{mM} \mathrm{NaCl}$ buffer, or $20 \%$ fetal calf serum in the same buffer. Values with reported error bars represent the average and standard deviations of measurements performed on at least three independently fabricated electrodes. Signal gain was computed by the relative change in SWV peak currents with respect to background current (SWV peak current in the absence of target). 

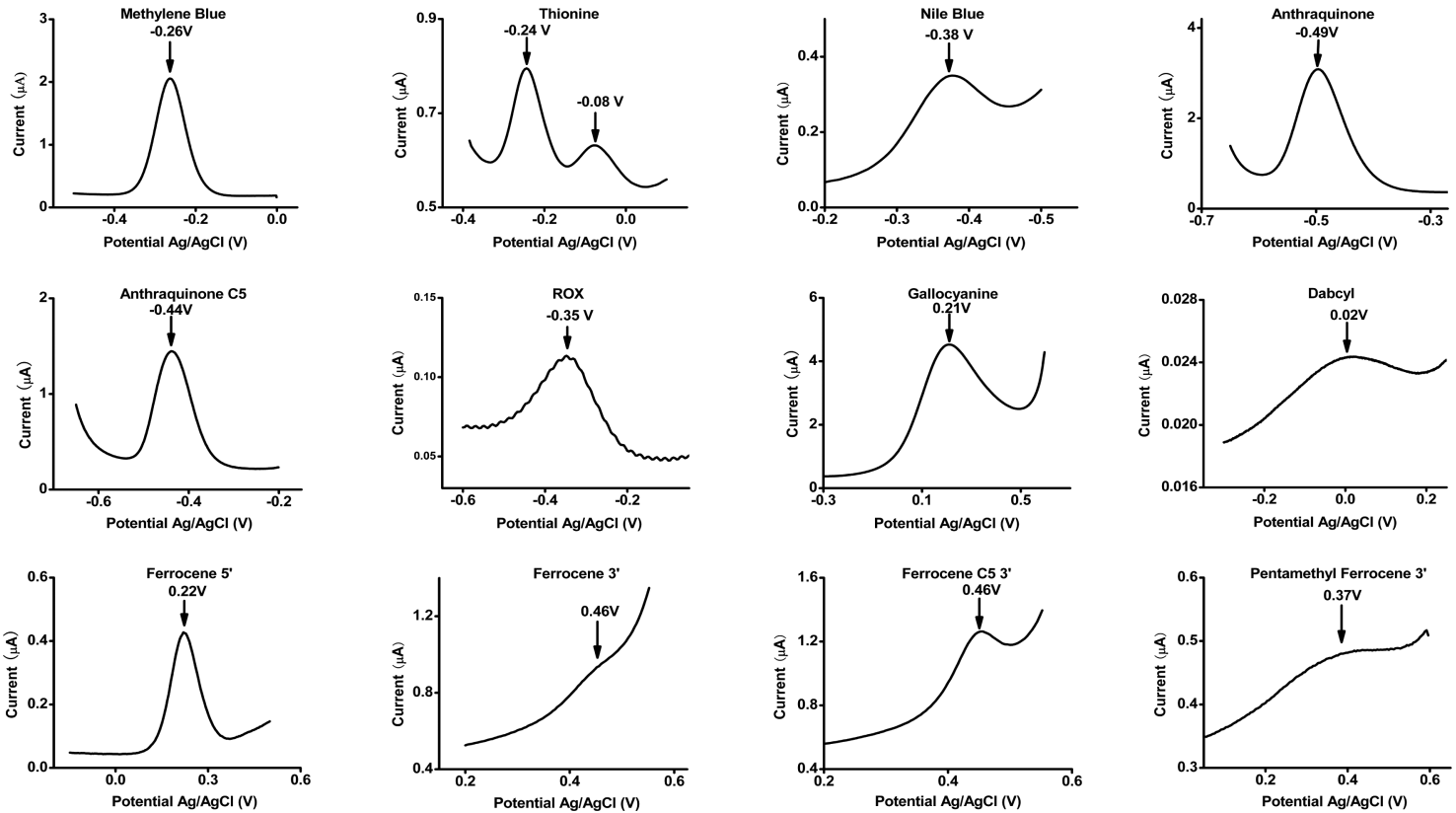

Figure 2. Not all redox reporters are created equal. Dabcyl and ROX, for example, fail to produce clear oxidation and reduction peaks when conjugated to DNA and interrogated using our standard square wave voltammetric parameters, and thionine exhibits two peaks in the relevant potential window. We investigated three ferrocene-containing constructs: one in which the ferrocene is conjugated directly on to an amine appended to the $5^{\prime}$ end of the DNA, a second in which the ferrocene is conjugated directly on to an amine appended to the $3^{\prime}$ end of the DNA, and a third, ferrocene C5, in which there is an additional spacer between ferrocene and the amide linkage to the DNA. The highly sloping baselines observed at potentials below $-0.5 \mathrm{~V}$ and above $0.5 \mathrm{~V}$ (vs Ag/AgCl) are due to the reduction of oxygen and the subsequent generation of reactive oxygen species (at low potentials) and the oxidation of gold (at high potentials). These same effects cause significant degradation of the thiol-on-gold SAM; that is, some redox reporters fail because they, themselves fail, and others fail because they report at potentials at which SAM stability is poor.

Our sensors were stored in buffer in sealed at room temperature. To test sensor robustness to repeated electrochemical interrogations, sensors were subjected to multiple SWV scans without target using a scan interval of 30 s in both buffer and $20 \%$ serum. To test sensor robustness against reuse we subjected them to repeated cycles of challenge with saturating target and regeneration (with a $30 \mathrm{~s}$ deionized water rinse). These cycles were repeated five times in $20 \%$ serum, respectively.

\section{RESULTS AND DISCUSSION}

The commercially available or easily synthetically accessible redox reporters we have characterized fall into either of two classes: organic small molecules and organometallic complexes. Among the former we have characterized methylene blue, thionine, anthraquinone, anthraquinone with a five-carbon linker (anthraquinone-C5), gallocyanine, Nile blue, indophenol, neutral red, dabcyl, and carboxy-X-rhodamine (ROX; Figure 1). Among the latter, we have characterized ferrocene attached directly to either the $5^{\prime}$ or $3^{\prime}$ end of the DNA, ferrocene linked to the DNA via a five-carbon linker (ferrocene-C5), and pentamethylferrocene. All told we have, over more than a decade of working in the field of electrochemical biosensors, characterized more than a dozen potential redox reporters, each as with a carboxylic acid group supporting ready conjugation to an amine-terminated DNA. Here we summarize the results of our many years' experience working with these potentially promising reporters.

To characterize the utility of these commercially or easily synthetically available redox reporters, we have over a number of years of research incorporated each into a simple E-DNA electrochemical DNA sensor. Specifically, we employed E-DNA sensors composed of linear DNA strands modified with the relevant redox reporter on its $3^{\prime}$-terminus (except for ferrocene, for which we have explored two $3^{\prime}$ and one $5^{\prime}$ linkage) and attached at its opposite terminus to a six-carbon alkanethiol monolayer to a gold electrode. ${ }^{23}$ Hybridization with a target oligonucleotide reduces the efficiency with which the attached redox reporter transfers electrons to the interrogating electrode, leading to a significant decrease in faradaic current when the system is interrogated using, for example, square wave voltammetry. For each of the resulting sensors, we measured signaling (i.e., do we see a clear, single oxidation and reduction peaks for the DNA-reporter conjugate), signal gain (relative signal change upon target saturation), electrochemical reversibility (i.e., its stability against multiple voltammetric scans), and stability when exposed to a realistically complex sample matrix (20\% blood serum).

The properties of the potential reporters we have characterized vary widely. For example, three of the potential reporters, thionine, dabcyl, and ROX, failed to produce clear, single oxidation and reduction peaks when conjugated to DNA (Figure 2a). A fourth potential reporter, indophenol, proved difficult for conjugation to DNA. Specifically, although free indophenol carboxylic acid is stable (and electrochemically active) under basic conditions we found that it decomposed during our attempts to conjugate it to an amine-terminated DNA. The redox potential of a fifth candidate, neutral red, shifts to $-0.7 \mathrm{~V}$ when modified to support DNA conjugation, moving its potential close to the redox potential of alkanethiols on gold and rendering the resultant sensor unstable (data not shown). Finally, a sensor employing a $3^{\prime}$ ferrocene reporter produced only a very small peak current (Figure 2) and, thus, we decide not to carry it over to our next testing step. The 
remaining reporters, in contrast, are all easily conjugated to amine-modified DNA and produce clear redox peaks within the potential window over which alkane-thiol-on-gold selfassembled monolayers are stable.

All of the remaining redox-reporters in our set produce at least reasonably high-gain E-DNA sensors under the standard volammetric parameters we employ in our sensors (Figure 3).

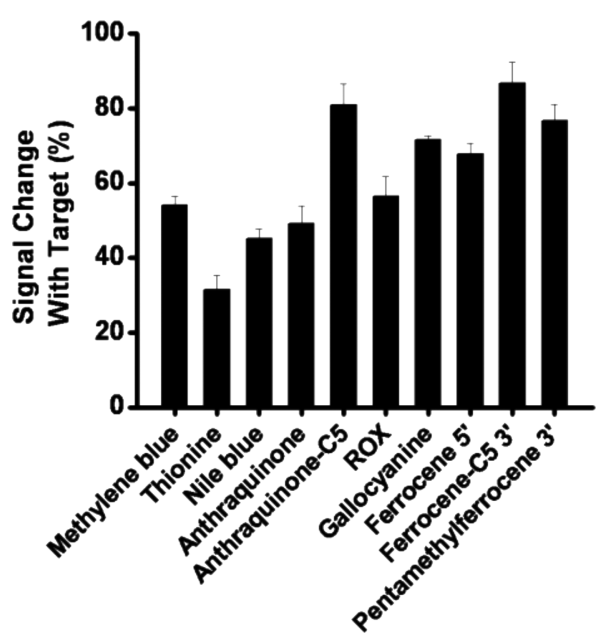

Figure 3. We challenged the reporter-conjugated probes that exhibit clear reduction and oxidation peaks with saturating concentrations of their complementary target to test their signaling properties. All respond as expected to these targets, albeit with varying signal gain. Of note signal gain in this class of sensors is a strong function of both the intrinsic electron transfer rate of the reporter and the square wave frequency and amplitude, thus, likely accounting for the variations in signal gain observed (all data were collected under a single set of square wave parameters).

The stability of the resultant sensors to repeated oxidationreduction cycles, however, varies widely (Figure 4). Sensors fabricated with methylene blue, for example, are impressively stable, exhibiting only $2 \%$ current loss after 100 square-wave voltammetric scans in buffer. Sensors employing anthraquinone, Nile blue, or 5'-linked ferrocene, in contrast, are only modestly stable under these conditions, exhibiting $~ 50 \%$ signal loss after 100 scans. Sensors fabricated using 3'-linked ferrocene, ferrocene-C5, and penta-methyl ferrocene linked are still less stable, losing $\sim 50 \%$ of their initial signal after only 50 scans. For most of the sensors we observe exhibit a steady, monotonic decrease in current as the number of scans increases. The signaling current of anthraquinone-based sensors, however, drops off rapidly during the first few scans before largely leveling off, and the signaling current from the gallocyanine-based sensor increases significantly upon repeated scanning. The origins of this increase are unknown to us.

An advantage of E-DNA-type sensors is their excellent performance in complex sample matrices, such as blood serum. ${ }^{1}$ Thus, motivated we have tested the performance of sensors fabricated using the reporters methylene blue, $5^{\prime}$-ferrocene, anthraquinone, and Nile blue when deployed in $20 \%$ blood serum. The gain of each is effectively indistinguishable from that seen in simple buffer solutions (Figure 5a). And, once again, we find that methylene blue is quite stable, exhibiting only minor signal loss when scanned twice an hour for more than $8 \mathrm{~h}$ under these conditions (Figure 5b). Sensors employing 5 '-ferrocene, anthraquinone (see, also, Figure SI 2 ), or Nile blue, in contrast, lose $25-30 \%$ of their original (a)

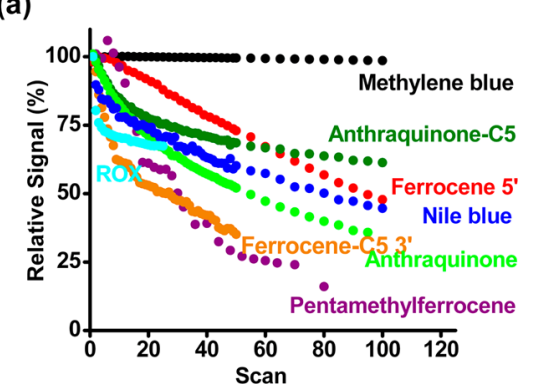

(b)

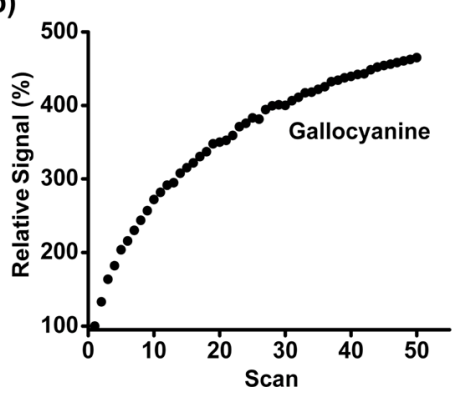

Figure 4. With the notable exception of methylene blue, (a) the redox reporters we have investigated are at least somewhat unstable against repeated voltammetric scans, here conducted in phosphate $/ \mathrm{NaCl}$ buffer. (b) Unlike the other reporters we have characterized, the signaling current from gallocyanine-based sensors increases upon repeated scanning. The scans in these figures are repeated every $20 \mathrm{~s}$, and thus the $x$-axis are also proportional to time. We find, however, that sensor decay (and, moreover, the differences in the decay rate from one reporter to the next) are a much stronger function of the number of scans than of time (data not shown).

signal under these same conditions. Finally, methylene bluebased sensors exhibit only $11 \%$ decay over four cycles of deployment in serum followed by regeneration (Figure 5c). Nile blue-based sensors, in contrast, exhibit $20 \%$ loss and anthraquinone- and ferrocene-based sensors exhibit about 50\% loss under these same use-and-regeneration conditions.

The origin of the loss in signal for most reporters is not clear, but investigation of their voltammograms and of prior literature descriptions of their chemistry provides some suggestions. Some of the degradation is, no doubt, due to the poor stability of some of the potential reporters. The ferrocenium ion, for example, is known to be unstable in aqueous solution in the presence of oxygen ${ }^{24}$ and the increasing current seen with gallocyanine presumably occurs due to redox-driven modification of the reporter. In addition, some of the degradation we see is likely due to the instability of the SAM when repeatedly scanned at high or low potentials. Specifically, a plot of stability (as measured by the current remaining after 50 scans) versus the redox potential of the reporter suggests that the best redox reporters all fall near the middle of the approximately $1 \mathrm{~V}$ window over which thiol-on-gold SAMs are stable (Figure 6). Moreover, this same plot illustrates an important point: there is a dearth of potential reporters in what appears to be the "sweet spot" in this plot (near $0 \mathrm{~V}$ vs $\mathrm{Ag} / \mathrm{AgCl}$ ), suggesting that efforts aimed at filling this gap may prove fruitful.

\section{CONCLUSIONS}

Here we have shown that, although a number of redox active moieties that are available in forms easily conjugated to DNA also support high-gain E-DNA signaling, the stabilities of the 
(a)

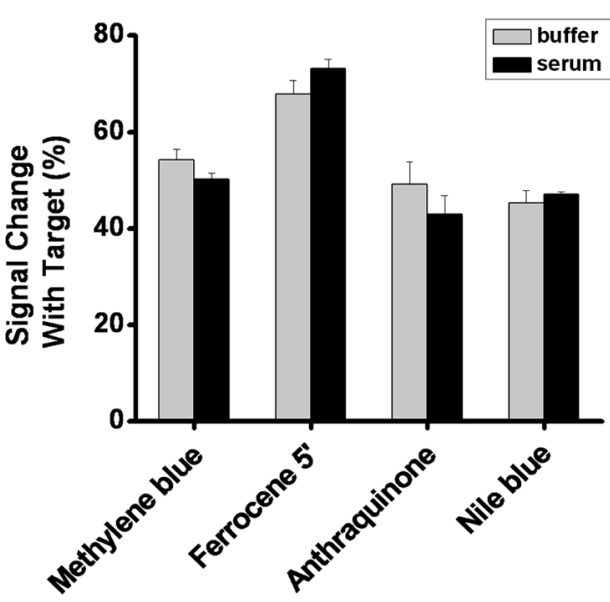

(b)

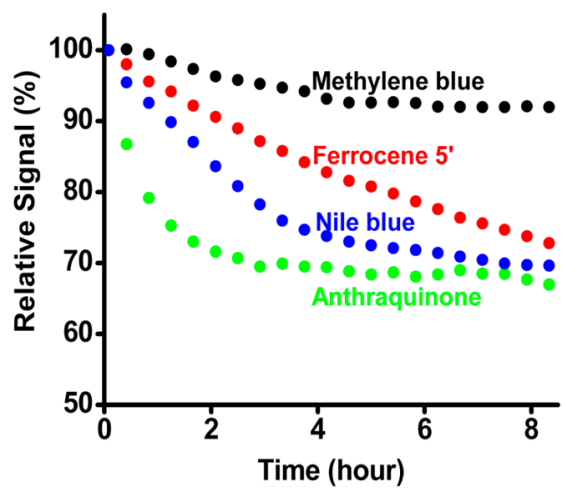

(c)

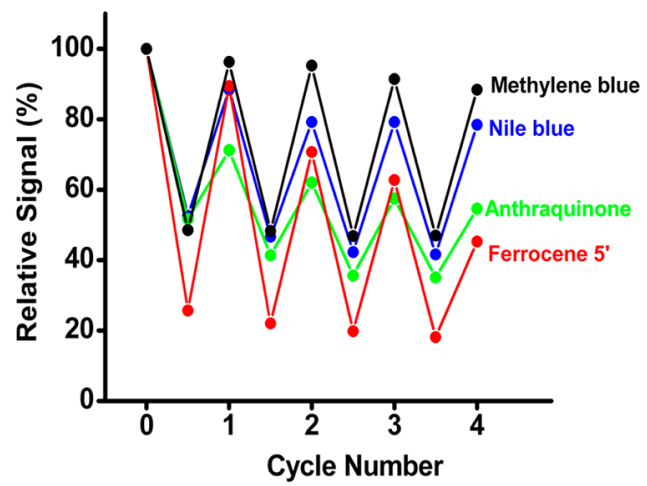

Figure 5. (a) Sensors fabricated with methylene blue, ferrocene, anthraquinone, or Nile blue exhibit similar signal gain in response to target binding whether deployed in simple buffer solutions or in $20 \%$ blood serum. (b) They all drift significantly, however, when repeatedly scanned in $20 \%$ serum over the course of hours, with methylene blue exhibiting the least drift. (c) Methylene blue-based sensors are likewise the most stable when the sensors are challenged with multiple cycles of hybridization (with saturating target) and regeneration (via diwater wash) in $20 \%$ blood serum.

resulting sensors differ dramatically due either to the inherent instability of the redox reporter or the instability of thiol-ongold SAMs at potentials below $-0.6 \mathrm{~V}$ or above $+0.4 \mathrm{~V}$ (vs Ag/ $\mathrm{AgCl}$ ). Sensors employing methylene blue, for example, exhibit outstanding stability even in blood serum, a complex, multicomponent, sample matrix. The next best reporters, in contrast, including ferrocene, anthraquinone, and Nile blue, also produce high-gain sensors but exhibit rather poorer

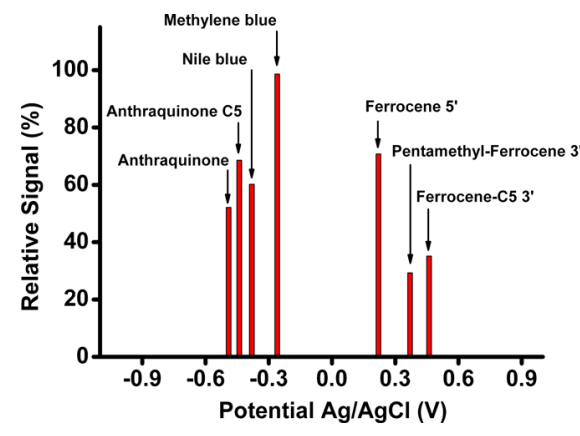

Figure 6. Plot of sensor stability (here measured as relative peak current remaining after 50 voltammetric scans) vs the redox potential of the reporter suggests that reporters situated near the middle of the $\sim 1 \mathrm{~V}$ window over which thiol-on-gold SAMs are stable exhibit the best performance.

stability against repeated voltammetric scanning at the relevant redox potentials. In addition to highlighting the advantages of employing methylene blue in such sensors, our results also suggest that more effort toward the development of suitable redox reporters is needed if the multiplexing and ratiometric error correction potential of electrochemical biosensors are to be fully realized.

\section{ASSOCIATED CONTENT}

\section{Supporting Information}

The Supporting Information is available free of charge on the ACS Publications website at DOI: 10.1021/acs.analchem.6b02376.

Analytical details and supporting figures (PDF).

\section{AUTHOR INFORMATION}

\section{Corresponding Author}

*E-mail: kwp@chem.ucsb.edu.

\section{Author Contributions}

The manuscript was written through contributions of all authors. All authors have given approval to the final version of the manuscript.

\section{Notes}

The authors declare no competing financial interest.

\section{ACKNOWLEDGMENTS}

This work was supported by the NIH (AI107936) and the Institute for Collaborative Biotechnologies through Grant W911NF-09-0001 from the U.S. Army Research Office (K.W.P.). The content of the information does not necessarily reflect the position or the policy of the Government, and no official endorsement should be inferred.

\section{REFERENCES}

(1) Lubin, A. A.; Plaxco, K. W. Acc. Chem. Res. 2010, 43, 496-505.

(2) McQuistan, A.; Zaitouna, A. J.; Echeverria, E.; Lai, R. Y. Chem. Commun. 2014, 50, 4690-4692.

(3) Zuo, X.; Xiao, Y.; Plaxco, K. W. J. Am. Chem. Soc. 2009, 131, 6944-6945.

(4) Boon, E. M.; Salas, J. W.; Barton, J. K. Nat. Biotechnol. 2002, 20, 282-286.

(5) Cash, K. J.; Ricci, F.; Plaxco, K. W. J. Am. Chem. Soc. 2009, 131, 6955-6957.

(6) Ikeda, R.; Kitagawa, S.; Chiba, J.; Inouye, M. Chem. - Eur. J. 2009, $15,7048-7051$. 
(7) Lubin, A. A.; Lai, R. Y.; Heeger, A. J.; Plaxco, K. W. Anal. Chem. 2006, 78, 5671-5677.

(8) Ferapontova, E. E.; Olsen, E. M.; Gothelf, K. V. J. Am. Chem. Soc. 2008, 130, 4256-4258.

(9) Mahshid, S. S.; Camiré, S.; Ricci, F.; Vallée-Bélisle, A. J. Am. Chem. Soc. 2015, 137, 15596-15599.

(10) Ferguson, B. S.; Hoggarth, D. A.; Maliniak, D.; Ploense, K.; White, R. J.; Woodward, N.; Hsieh, K.; Bonham, A. J.; Eisenstein, M.; Kippin, T.; Plaxco, K. W.; Soh, H. T. Sci. Transl. Med. 2013, 5, 213 ral65.

(11) Xiao, Y.; Lai, R. Y.; Plaxco, K. W. Nat. Protoc. 2007, 2, 28752880.

(12) Rowe, A. A.; Bonham, A. J.; White, R. J.; Zimmer, M. P.; Yadgar, R. J.; Hobza, T. M.; Honea, J.; Ben-Yaacov, I.; Plaxco, K. W. PLoS One 2011, 6, e23783.

(13) Kang, D.; Xia, F.; Zuo, X.; White, J. R.; Vallée-Bélisle, A.; Plaxco, K. W. NPG Asia Mater. 2012, 4, e1.

(14) Xia, J.; Daimin, S.; Wang, Z.; Zhang, F.; Yang, M.; Gui, R.; Xia, L.; Bi, S.; Xia, Y.; Li, Y.; Xia, L. Biosens. Bioelectron. 2015, 68, 55-61.

(15) Du, Y.; Lim, B. J.; Li, B.; Jiang, Y. S.; Sessler, J. L.; Ellington, A. D. Anal. Chem. 2014, 86, 8010-8016.

(16) Ren, K.; Wu, J.; Yan, F.; Zhang, Y.; Ju, H. Biosens. Bioelectron. 2015, 66, 345-349.

(17) Fan, C.; Plaxco, K. W.; Heeger, A. J. Proc. Natl. Acad. Sci. U. S. A. 2003, 100, 9134-9137.

(18) Gorodetsky, A. A.; Hammond, W. J.; Hill, M. G.; Slowinski, K.; Barton, J. K. Langmuir 2008, 24, 14282-14288.

(19) Pheeney, C. G.; Barton, J. K. J. Am. Chem. Soc. 2013, 135, 14944-14947.

(20) Balintová, J.; Pohl, R.; Horáková, P.; Vidláková, P.; Havran, L.; Fojta, M.; Hocek, M. Chem. - Eur. J. 2011, 17, 14063-14073.

(21) Zuo, X.; Song, S.; Zhang, J.; Pan, D.; Wang, L.; Fan, C. J. Am. Chem. Soc. 2007, 129, 1042-1043.

(22) Kang, D.; Zuo, X.; Yang, R.; Xia, F.; Plaxco, K. W.; White, R. J. Anal. Chem. 2009, 81, 9109-9113.

(23) Ricci, F.; Lai, R. Y.; Heeger, A. J.; Plaxco, K. W.; Sumner, J. J. Langmuir 2007, 23, 6827-6834.

(24) Singh, A.; Chowdhury, D. R.; Paul, A. Analyst 2014, 139, 57475754. 